\title{
Graphene sensing an inhomogeneous strain due to the surface relief in FeNiCoTi shape memory alloy
}

\author{
Liqiang Zhang, ${ }^{\text {a,bt }}$ Yang Shao, ${ }^{\text {a,bt }}$ Zhiqiang Tu, ${ }^{\text {at }}$ Rui Liu, $^{c}$ Fan Yang, \\ Daqiang Jiang, ${ }^{a, b}$ Yunpeng Guo, ${ }^{a, b}$ Zhizhen Ye, ${ }^{d}$ Ting Liu, ${ }^{a}$ \\ Junsong Zhang, ${ }^{a, b}$ Zhenfei Gao, ${ }^{a, b}$ Yongfeng $\mathrm{Li}^{\mathrm{a} *}$ and Lishan Cui ${ }^{\mathrm{a}, \mathrm{b}_{*}}$
}

In this paper, we present a novel method of using graphene for sensing the inhomogeneous strain due to the surface relief in FeNiCoTi shape memory alloy. In the experiment, a large sheet of graphene fabricated by chemical vapor deposition was transferred onto the FeNiCoTi substrate. The flat surface of the substrate would become wrinkled due to the surface relief formed during the FeNiCoTi substrate phase transformation, meanwhile loading a tensile strain on the surface graphene. It is found that the 2D Raman peak of graphene demonstrates a significant red shift due to the tensile strain. The different colors exhibited in the Raman mapping image of the graphene directly displayed the strain distribution information across the surface. In the future, we may alter to quantitatively analyze the surface relief by using Raman spectroscopy instead of the atomic force microscopy. Copyright @ 2013 John Wiley \& Sons, Ltd.

Additional supporting information may be found in the online version of this article at the publisher's web site.

Keywords: graphene; sensing; inhomogeneous; strain; shape memory alloy

\section{Introduction}

Graphene, a closely stacked two-dimensional (2D) honeycomb of $S P^{2}$-peaked carbon atoms, is an extremely interesting material since its discovery by Geim et al. in $2004{ }^{[1,2]}$ It has been found that monolayer graphene exhibit many extraordinary properties, including ultrahigh thermal conductivity $(5300 \mathrm{~W} / \mathrm{m} \mathrm{K}),{ }^{[3]}$ ultrahigh electron mobility (over $15,000 \mathrm{~cm}^{2} / \mathrm{Vs}$ ), ${ }^{[4]}$ ultralow resistivity $\left(10^{-6} \Omega \mathrm{cm}\right){ }^{[5]}$ high specific surface area $\left(2630 \mathrm{~m}^{2} / \mathrm{g}\right){ }^{[6]}$ high strength $(110 \mathrm{GPa}){ }^{[7]}$ etc. ${ }^{[8-11]}$ Because of its so many outstanding properties, graphene holds great promise for widespread applications such as field-effect transistors, ${ }^{[6]}$ sensors, $^{[12]}$ solar cells, ${ }^{[5]}$ nanocomposites, ${ }^{[13]}$ super-capacitors, ${ }^{[14]}$ etc. ${ }^{[15-17]}$ Among its plenty of potential applications, graphene-based strain sensors are attracting more and more attention, ${ }^{[18-20]}$ which can be used to detect the physical deformations or structural changes. ${ }^{[21]}$ Lee et al. have pointed out that graphene behaves a sensitive response to the strain with a gage factor of $6.1 .^{[22]}$ Bae et al. fabricated a graphene strain sensor on a flexible plastic substrate, and they investigated its piezoresistive property under a tensile strain. ${ }^{[23]}$ As we have known, Raman spectroscopy is a good tool for probing the mechanical properties of nanomaterials. ${ }^{[24]}$ It has been found that the intervalley two phonon mode (2D), which involves scattering of electron-hole pairs between neighboring Dirac cones, is particularly sensitive to the strain changes. ${ }^{[25]}$ Therefore, most researchers use the 2D peak shift in Raman spectrum to investigate the strain loaded on graphene. ${ }^{[26]} \mathrm{Ni}$ et al. applied a uniform tensile strain on graphene by stretching the polyethylene terephthalate substrate, and they observed a significant red shift of the Raman 2D peak $\left[27.8 \mathrm{~cm}^{-1}\right.$ per $1 \%$ strain (/\%)] and $G$ peak $\left(14.2 \mathrm{~cm}^{-1} / \%\right)$ for the monolayer graphene. ${ }^{[27]}$
Tsoukleri et al. also observed a large variation of the graphene 2D peak with strain at a rate higher than $\sim 50 \mathrm{~cm}^{-1}$ under $1 \%$ strain. ${ }^{[28]}$

Until now, enormous efforts are being devoted to investigate the strain influence on graphene. ${ }^{[29-33]}$ However, there are few reports on detecting a strain by using graphene in a practical application. As we have known, the flat surface of the shape memory alloy (SMA) in austenite will become rough upon cryogenic treatment, and this interesting phenomenon is called surface relief. ${ }^{[34]}$ After SMA phase transformation, its surface will become wrinkled, meanwhile forming an inhomogeneous tensile strain on the surface. In the past, only the atomic force microscopy (AFM) was employed to investigate the surface relief. ${ }^{[35,36]}$

* Correspondence to: Yongfeng Li, State Key Laboratory of Heavy Oil Processing, China University of Petroleum, Beijing 102249, China.

E-mail: yfli@cup.edu.cn

* Correspondence to: Lishan Cui, Department of Materials Science and Engineering, China University of Petroleum, Beijing 102249, China.

E-mail: lishancui63@126.com

${ }^{+}$L.Q. Zhang, Y. Shao. and Z.Q. Tu contributed equally to this work.

a State Key Laboratory of Heavy Oil Processing, China University of Petroleum, Beijing 102249, China

b Department of Materials Science and Engineering, China University of Petroleum, Beijing 102249, China

c State Key Laboratory of Environmental Chemistry and Ecotoxicology, Research Center for Eco-Environmental Sciences, Chinese Academy of Sciences, Beijing 100085, China

d State Key Laboratory of Silicon Materials, Department of Materials, Zhejiang University, Hangzhou 310027, China 
However, an AFM image can merely display the surface roughness; how to quickly detect the inhomogeneous strain distribution across the wrinkled surface after phase transformation is still an unsolved issue.

Thus, in this study, we develop a method of sensing the inhomogeneous strain due to surface relief in FeNiCoTi SMA by using graphene. In the experiment, a large sheet of monolayer graphene fabricated by chemical vapor deposition (CVD) was transferred onto the FeNiCoTi SMA substrate. After FeNiCoTi SMA phase transformation, the flat surface of the pristine substrate became covered with significant relief, during which loading an inhomogeneous tensile strain on the surface graphene. It is found that the strain distribution information was successfully printed on the graphene, which can be quickly detected by analyzing the 2D peak shifts of graphene in its Raman spectrum. Moreover, the relief angle of the surface relief can also be easily calculated from the Raman mapping image of the strained graphene.

\section{Experimental section}

\section{Synthesis of monolayer graphene by a CVD method}

Monolayer graphene films were grown on $25-\mu \mathrm{m}$-thick copper foils (Alfa Aesar, item No.046365, cut into $2 \times 2$-cm square plates) using high-vacuum CVD in a 4-inch chamber quartz reactor. The CVD used in this study is an ordinary thermal hot-walled low pressure CVD, which was produced by Tianjin Central Furnace Company (K-G08123K). Prior to the experiment, the copper foils were first immersed in 5\% aqueous acetic acid solution for $5 \mathrm{~min}$, and then ultrasonically cleaned with acetone for $5 \mathrm{~min}$, and finally dried under nitrogen atmosphere.

Firstly, the cleaned copper foils were sent to the center of the chamber and then high-purity hydrogen (99.999\%) and argon (99.999\%) were introduced into the chamber at a speed of $50 \mathrm{sccm} / \mathrm{min}$ and $300 \mathrm{sccm} / \mathrm{min}$, respectively. The silica tube was heated up to $1000^{\circ} \mathrm{C}$ during graphene synthesis accompanied with introducing $35 \mathrm{sccm} / \mathrm{min}$ of $\mathrm{CH}_{4}$ for $8 \mathrm{~min}$. The purity of the $\mathrm{CH}_{4}$ is $99.9 \%$, and the deposition pressure is 400 mTorr. After this step, $\mathrm{CH}_{4}$ was cut off and the furnace was cooled down to room temperature under flowing $300 \mathrm{sccm} / \mathrm{min}$ of $\mathrm{Ar}$ and $35 \mathrm{sccm} / \mathrm{min}$ of $\mathrm{H}_{2}$. The heating rate was $12^{\circ} \mathrm{C} / \mathrm{min}$, whereas the cooling rate was $10^{\circ} \mathrm{C} / \mathrm{min}$.

\section{The processes of transferring the monolayer graphene onto the FeNiCoTi SMA substrate by a CVD method}

Monolayer graphene films were obtained by etching $\mathrm{Cu}$ foils in an aqueous solution of ferric chloride. Typically, before etching, the graphene-on-Cu was coated with poly-methyl methacrylate (PMMA). After the etching process, it was transferred by silicon wafers to deionized water. The PMMA/graphene stack was then lifted by the target substrate. In order to smooth the wrinkles caused during the transfer process, ethanol was sprayed to the surface of the stack leading to a full contact between PMMA/ graphene and substrate. The drying process was performed at $50^{\circ} \mathrm{C}$ in an oven for about $3 \mathrm{~h}$ to vaporize the water molecules. Afterwards, acetone was introduced to dissolve the PMMA. Finally, the monolayer graphene was successfully transferred onto the FeNiCoTi substrate.

\section{The method of fabricating the FeNiCoTi SMA substrates}

FeNiCoTi SMA ingots used for fabricating the FeNiCoTi SMA substrates were prepared by using Fe-31\%Ni-10\%Co-4\%Ti (at.\%), and all metals have a high purity above $99.9 \%$. The alloy ingot of FeNiCoTi was melted by vacuum induction and then it was hotrolled into cylindrical bars, and hot-forged into rods with $12 \mathrm{~mm}$ in diameter. The rods were subsequently annealed at $1473 \mathrm{~K}$ for $20 \mathrm{~min}$, after which they were quenched to room temperature to obtain austenite. The FeNiCoTi SMA substrates were obtained by cutting the rods along their axial direction by a discharge wire cutting method with $\Phi 12 \times 2 \mathrm{~mm}$. After being polished, the FeNiCoTi SMA substrates are ready to be used for transferring graphene.

\section{The processes of realizing the FeNiCoTi SMA substrate phase transformation and producing surface relief}

In order to realize the FeNiCoTi SMA substrate phase transformation and produce surface relief, the samples were deep cryogenically treated by dipping into the cooling liquid; during which, an inhomogeneous strain on the graphene was loaded. The cooling liquid $\left(-60^{\circ} \mathrm{C}\right)$ was tuned by mixing liquid nitrogen and alcohol with a volume ratio of $1: 2$, which is below the FeNiCoTi SMA martensitic phase transformation temperature.

\section{The characterizations of the FeNiCoTi SMA substrates and graphene}

The phase transformation behavior was characterized by using Bede D1 X-ray diffraction (XRD) system with a $\mathrm{Cu} \mathrm{K} \alpha$ radiation $(\lambda=0.15406 \mathrm{~nm})$. Surface morphology was studied by using the FEI Quanta 200 F scanning electron microscope (SEM) operated at a voltage of $20 \mathrm{kV}$ and the Pico Scan TM 2500 AFM under a tapping mode. The layer number of graphene is identified by using the FEI Tecnai G2 F20 transmission electron microscopy (TEM), which is operated at $200 \mathrm{kV}$. The TEM analyses included the common TEM images at a low magnification, high-resolution TEM (HR-TEM) images and selected area electron diffraction (SAED) patterns. The microscopic Raman spectra were recorded by using a Micro-Raman spectrometer (InVia Reflex, Rensihaw, UK). The wavelength of the Raman excitation was $532 \mathrm{~nm}$, and the laser power was kept below $0.85 \mathrm{~mW}$ to avoid laser-induced local heating on the sample. All peaks in the Raman spectra were fitted with Lorentzians. A $100 \times$ objective lens with a numerical aperture of 0.95 was used in the Raman experiments, and the spot size was estimated to be about $500 \mathrm{~nm}$. For taking the Raman mapping image, the sample was placed on an $x-y$ piezostage and scanned point by point.

\section{Results and discussion}

Figure 1 shows the schematic of loading a tensile strain on graphene due to the FeNiCoTi SMA substrate surface relief after phase transformation. In the experiment, pristine monolayer graphene fabricated via a CVD process was firstly transferred onto the FeNiCoTi SMA substrate and then the sample was deep cryogenically treated. Obviously, surface relief due to temperature-induced martensitic transformation of the FeNiCoTi SMA substrate was formed upon deep cryogenic treatment, during which loading a tensile strain on the graphene. The microscope images of the graphene deposited on the FeNiCoTi SMA substrate before and after surface relief are compared in the insert 


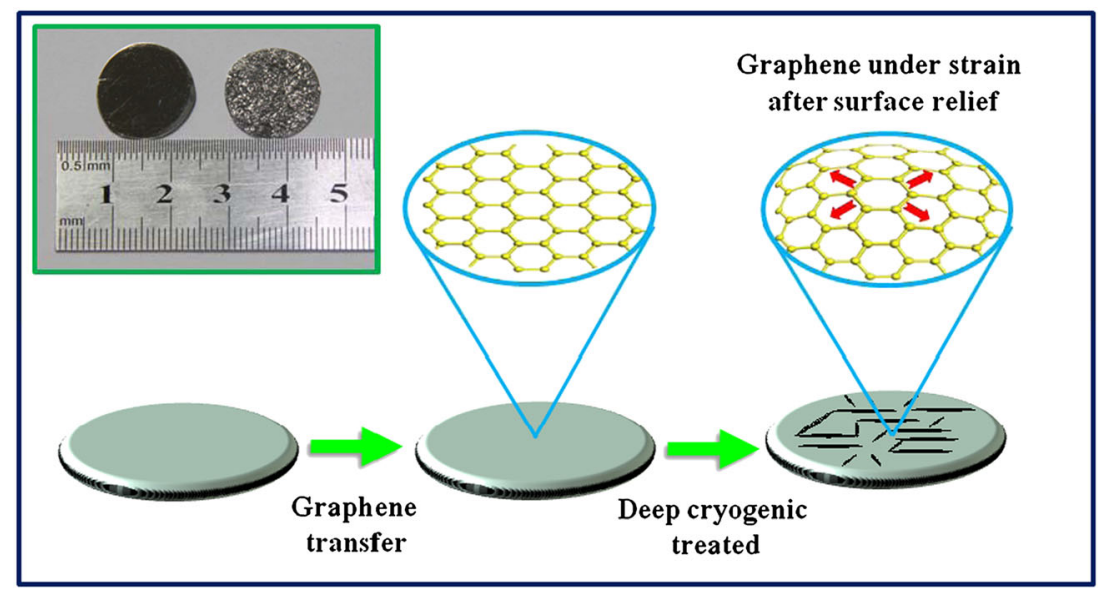

Figure 1. The schematic of loading a tensile strain on graphene due to the FeNiCoTi SMA substrate surface relief. Firstly, the graphene was transferred onto the FeNiCoTi SMA substrate and then the sample was upon deep cryogenic treatment to produce the surface relief. The inset compares the optical micrographs of the graphene coated FeNiCoTi SMA substrate before and after surface relief.

of Fig. 1. It shows that the pristine FeNiCoTi SMA substrate is flat and smooth. Because of mirror reflection, the pristine sample looks dark when taking a photo from the side. However, after deep cryogenic treatment, its surface became bright because of diffuse reflection, which originated from the wrinkles formed during the FeNiCoTi SMA substrate phase transformation.

Figure 2 shows the TEM image, SAED pattern, and the Raman spectrum of the graphene used in this study. The graphene sample prepared for TEM observation is the same as that transferred onto the FeNiCoTi SMA substrate. Figure 2(a) shows the graphene morphology in the TEM at a low magnification, in which a film-like graphene is observed. An HR-TEM image at the edge of the graphene gives the number of layers by direct visualization (Fig. 2(b)). Obviously, there is only one dark line at the edge, which indicates the monolayer structure of the graphene. ${ }^{[37]}$ Additionally, the SAED analysis (Fig. 2(c)) was employed to determine its number of layers, in which the six sharp points in the inner hexatomic ring are similar to the case of reported monolayer graphene. ${ }^{[37]}$ Furthermore, the relative intensities of the diffraction spots (2) and (3) (inner hexagon) are nearly twice as high as those of spots (1) and (4) (outer hexagon), as shown in Fig. 2(d), which further confirmed that our graphene is monolayer. ${ }^{[37]}$ In addition, Raman spectrum is also measured on graphene (Fig. 2(e)). For the Raman study, we directly used the graphene sample transferred onto the surface of the FeNiCoTi SMA substrate. There are two prominent peaks in the Raman spectrum, one is around $\sim 1581 \mathrm{~cm}^{-1}$, commonly called the $G$ peak, which originated from the Raman active $E_{2 g}$ phonon (in-plane optical mode). ${ }^{[38]}$ The other is located at $\sim 2689 \mathrm{~cm}^{-1}$, normally called the 2D peak, which is due to the second order of zone-boundary phonons. ${ }^{[38]}$ Here, we found a single, sharp $\left(\sim 42 \mathrm{~cm}^{-1}\right)$ 2D peak in graphene, which has the typical characteristic of monolayer graphene as reported in the literature. ${ }^{[37]}$ The HR-TEM image at the edge of graphene, the SAED pattern, and the Raman spectrum analysis provide strong evidence that the graphene used for this study is monolayer.
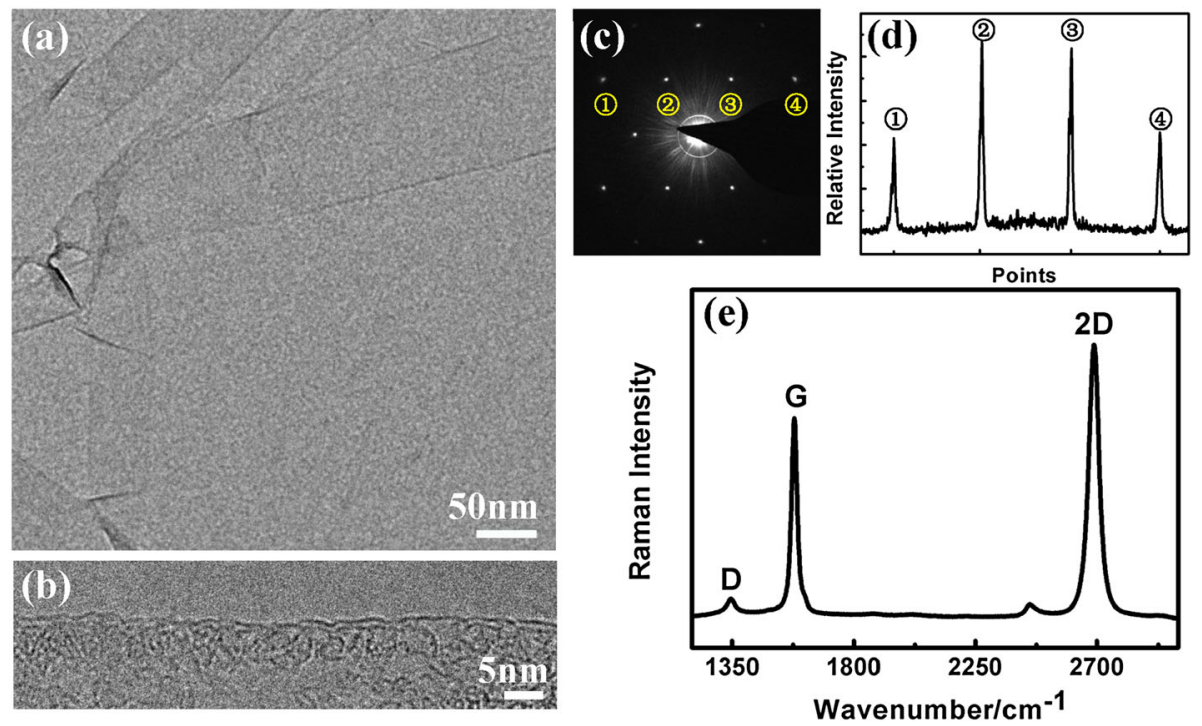

Figure 2. (a) The graphene morphology in the transmission electron microscopy at a low magnification. (b) A high-resolution transmission electron microscopy image of the graphene at the edge. (c) Selected area electron diffraction pattern of the graphene. (d) The relative intensities of the diffraction spots (1) and (4) (outer hexagon) and spots (2) and (3) (inner hexagon). (e) The $532 \mathrm{~nm}$ Raman spectrum of the graphene. 
The FeNiCoTi SMA substrate phase transformation behavior was characterized by using XRD, as shown in Fig. 3. SEM was employed to study the surface morphology changes after phase transformation, which is shown in the insert of Fig. 3. XRD results revealed that the pristine FeNiCoTi SMA substrate is in its austenite phase $(A)$, whereas it partly converted to martensite phase (M) after phase transformation. During this process, the intensity of (110)M peak related to the martensite of FeNiCoTi enhanced, whereas the intensity of peaks (200)A and (220)A related to its austenite phase demonstrated an obvious decrease, which is in agreement with the SEM characterization (the insert of Fig. 3). After substrate phase tranformation, the flat surface of the pristine sample became covered with significant relief morphology. It is necessary to note that as the phase transformation temperature of FeNiCoTi SMA is much lower than room temperature, the wrinkles due to the substrate surface relief can remain stable at room temperature.

As the Raman 2D peak of graphene exhibits a special sensitivity to the strain, it is expected to use the shift of its 2D peak to characterize the loaded strain after surface relief. Before carrying out the experiment, it was confirmed that the FeNiCoTi SMA substrate exhibited no typical Raman signals, so all the detected Raman singles can be attributed to vibrations of graphene on the substrate surface. As reported previously, ${ }^{[30]}$ even within the same piece of graphene, the wavenumber of the 2D mode could be slightly different from one region to the other. So in this study, ten points from both the pristine sample and the sample after phase transformation were randomly chosen to avoid the occurrence of errors, as shown in Figure S1 (Supporting Information), in which their corresponding 2D peak positions (bars) and peak widths (blue square) are compared. It is found that the loaded strain on graphene due to surface relief was strongly correlated with its 2D peak shift. All the 2D peaks of graphene demonstrate a significant red shift after phase transformation compared with the pristine sample, which confirms that the graphene is loaded by a tensile strain due to surface relief. However, the 2D peak

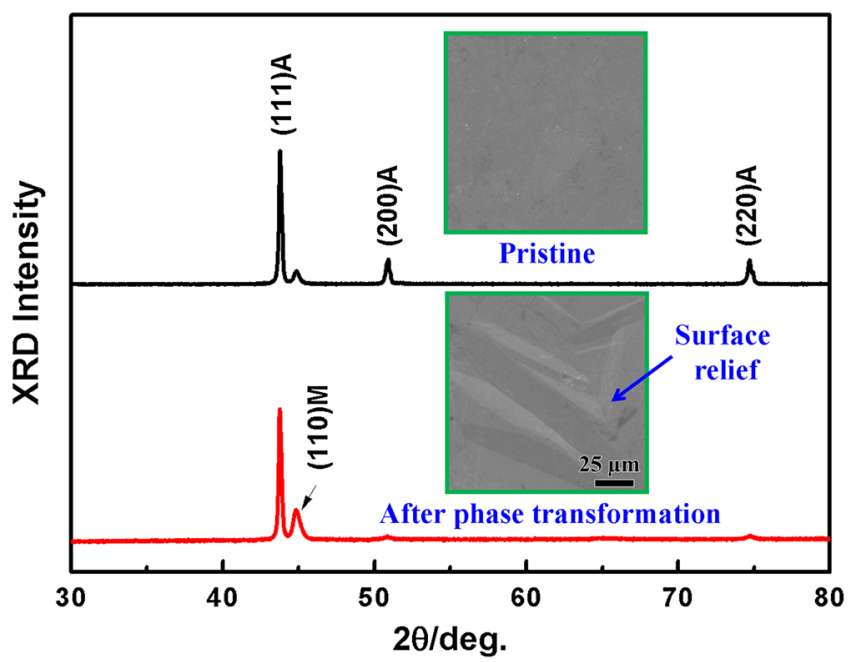

Figure 3. X-ray diffraction (XRD) and scanning electron microscope analysis of the pristine FeNiCoTi shape memory alloy substrate and its changes after phase transformation. The peaks (111)A, (200)A, and (220) $\mathrm{A}$ are related to the pristine FeNiCoTi in its austenite phase; the peak (110)M is related to the FeNiCoTi martensite phase after phase transformation. The insets compare the shape memory alloy surface morphology of FeNiCoTi before and after phase transformation, from which significant relief morphology can be seen after phase transformation. widths hardly demonstrate a distinguishable dependence on the strain. The red shift of the 2D peak can be understood on the basis of elongation of the carbon-carbon bonds, which weakens the bonds and therefore lowers their vibration wavenumber. In addition, the significant red shift of the 2D peaks indicates that the strain has been transferred well from the substrate deformation to the surface graphene.

In order to make a detailed understanding of the strain distribution across surface, it is necessary to take a Raman mapping of the graphene. Figure 4(a) demonstrates an optical micrograph of the sample after phase transformation, in which two main ridge areas of graphene are indicated by two circles and named ridge 1 and 2, respectively. The corresponding contour mapping of the 2D peaks for these two ridge areas is shown in Fig. 4(b), which was generated from the measured data of the 2D peak positions. It is obvious that the $2 \mathrm{D}$ peaks demonstrate fluctuated shifts across the surface, suggesting that the graphene was loaded with an inhomogeneous strain. It is found that the 2D peak of the pristine graphene is located at $\sim 2686 \mathrm{~cm}^{-1}$ on average according to the statistics shown in Figure S1. Because of the substrate surface relief, the 2D peak position around ridge 1 moves to $\sim 2675 \mathrm{~cm}^{-1}$ on the slope (green region) and shifts to $\sim 2664 \mathrm{~cm}^{-1}$ at the top (blue region), which is owing to that the vibration wavenumber of graphene is affected by an inhomogeneous strain.

In the past, only AFM can quantitatively analyze the surface relief, ${ }^{[35,36]}$ but in our case, the surface relief is altered to be quantitatively investigated by monitoring the graphene 2D peak shift directly. However, as the graphene Raman 2D band wavenumber shift rate due to strain is still unknown, AFM is still needed prior to the first experiment. Figure 5(a) shows the
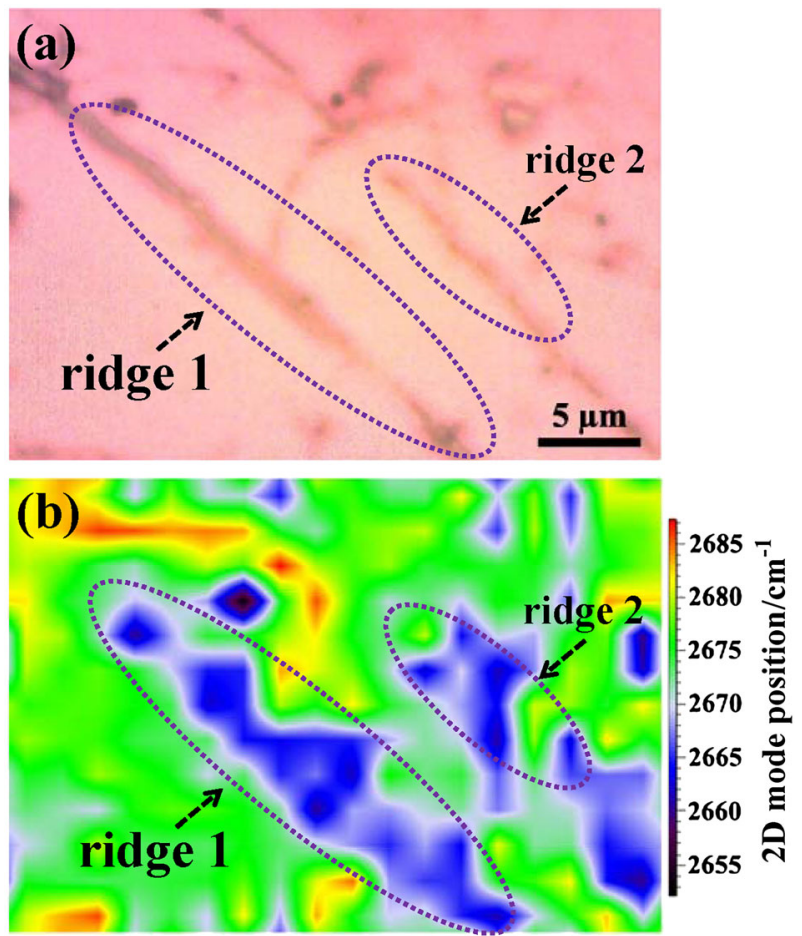

Figure 4. (a) The optical micrograph of the graphene on FeNiCoTi shape memory alloy substrate after phase transformation. (b) A contour mapping of the 2D peak of the graphene after substrate phase transformation at the same position. Two typical ridges on the wrinkle surface are marked by two circles, respectively. 

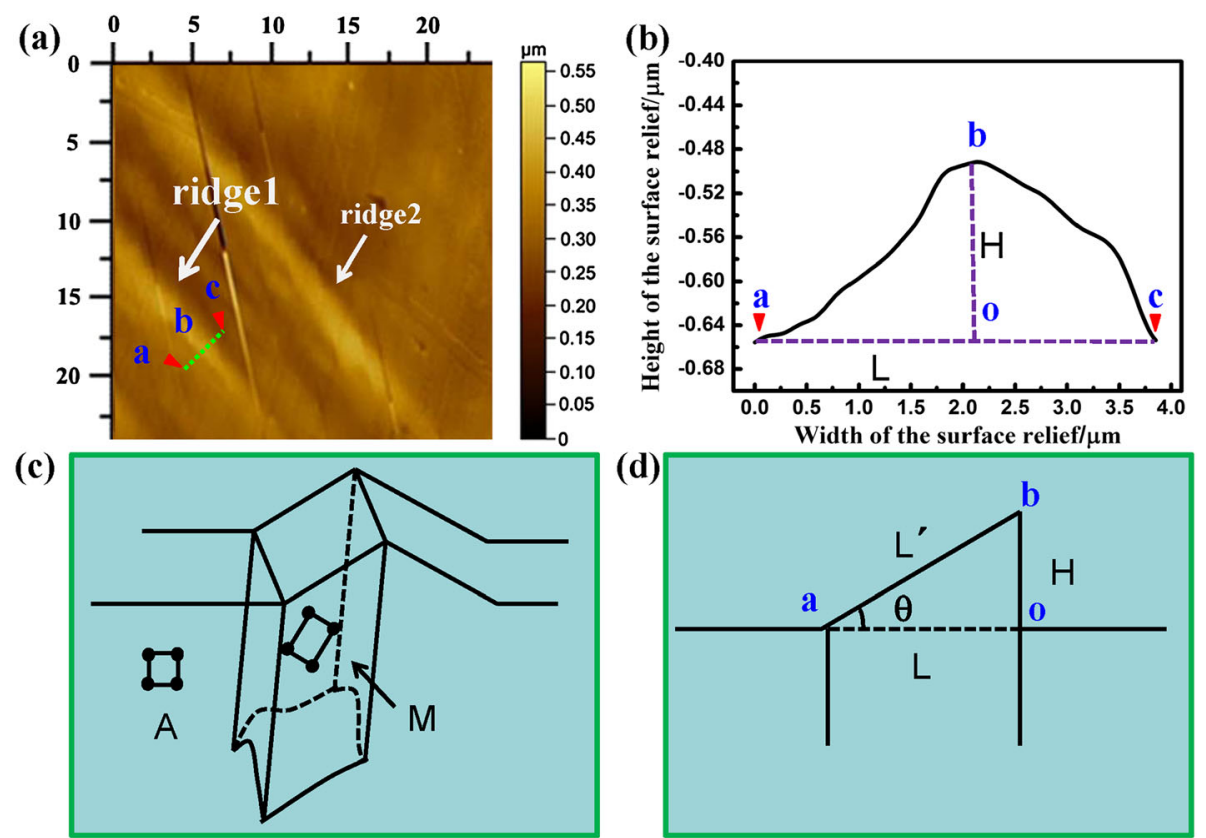

Figure 5. (a) The surface relief is quantitatively analyzed by atomic force microscopy. The position $a$, b, and c represent the bottom, top, bottom of the ridge 1, respectively. (b) A fluctuating curve is made alone the green line in (a). (c) The schematic illustration of martensitic surface relief due to the $\gamma(\mathrm{fcc}) \rightarrow \varepsilon(\mathrm{hcp})$ phase transformation. (d) The schematic illustration for calculating the relief angle. This figure is available in colour online at wileyonlinelibrary.com/journal/jrs

surface relief analyzed quantitatively by AFM after the substrate martensitic phase transformation. Figure $5(\mathrm{~b})$ shows a curve of height of surface relief scanning alone the green line in Fig. 5 (a). The positions $a, b$, and c represent the bottom, top, bottom of the ridge 1, respectively. Figure 5(c) shows the schematic illustration of surface relief due to the $\gamma(\mathrm{fcc}) \rightarrow \varepsilon$ (hcp) martensitic phase transformation. Figure $5(\mathrm{~d})$ is the schematic illustration for the relief angle which can be calculated by $\tan \theta=\mathrm{H} / \mathrm{L}$, where $\mathrm{H}$ and $\mathrm{L}$ are the height and width of surface relief, respectively. ${ }^{[35]}$ The calculated relief angle $\theta$ for the $\gamma(\mathrm{fcc}) \rightarrow \varepsilon(\mathrm{hcp})$ transformation is about $4.57^{\circ}$, corresponding to a tensile strain $\varepsilon$ of $0.32 \%$ by using the formula $\varepsilon=\left(\mathrm{L}^{\prime}-\mathrm{L}\right) / \mathrm{L}$. Therefore, as a rough estimation, the $2 \mathrm{D}$ peak wavenumber shift rate dependence on the strain is $\sim 31.2 \mathrm{~cm}^{-1} \%\left(31.2 \mathrm{~cm}^{-1}\right.$ per $1 \%$ strain), which is similar to that reported by $\mathrm{Ni}$ et al. on the red shift of the Raman 2D peak $27.8 \mathrm{~cm}^{-1} \%$ under uniaxial tensile strain. ${ }^{[27]}$ As the 2D peak shift rate of graphene is much larger than that of single well carbon nanotubes $\left(-7.9 \mathrm{~cm}^{-1}\right)$, multiwalled carbon nanotubes $\left(-0.48 \mathrm{~cm}^{-1}\right)$, and $C_{60}\left(-0.13 \mathrm{~cm}^{-1}\right)$, it is far superior in fabricating the strain sensor. ${ }^{[28]}$

Up to now, the surface relief can be absolutely quantitatively analyzed by monitoring the graphene $2 \mathrm{D}$ shift directly without using AFM. By referring with the $2 \mathrm{D}$ shift rate, it can be calculated that the strain value around the top of the ridge is about $0.71 \%$, which is roughly twice as that on the slope $(0.32 \%)$. Herein, we selected another ridge (ridge 2 ) for verification experiment, as shown Fig. 5(b). The 2D peak at the slope of ridge 2 demonstrates an obvious downshift of $10 \mathrm{~cm}^{-1}$ from 2686 to $2676 \mathrm{~cm}^{-1}$, which is close to that of ridge 1 . The loaded strain $\varepsilon$ on graphene was about $0.31 \%$ according to its 2D shift rate of $\sim 31.2 \mathrm{~cm}^{-1} \%$ obtained from the aforementioned discussion. Then, the relief angle $\theta$ can be calculated by using the formula $\theta=\operatorname{arcos}(1 / 1+\varepsilon)$, which is about $4.51^{\circ}$. The strain and relief angle information obtained by analyzing the graphene are consistent with the results obtained from AFM.
Compared with previous studies, we alter to quantitatively analyze the surface relief by using graphene instead of the traditional AFM. In the past, the strain loaded on graphene by stretching the polymer substrate or epitaxial growth are usually uniaxial and uniform, ${ }^{[27,28]}$ whereas in this study, an inhomogeneous tensile strain was successfully applied on graphene. Additionally, graphene exhibits its extraordinary sensitivity to the minor strain due to surface relief (almost below 1\% because of substrate surface relief). Moreover, although the phase transformation deformation speed is up to $\sim 10^{3} \mathrm{~m} / \mathrm{s}$, the graphene can still remain its integrity structure, which fully exhibits the perfect mechanical properties of the graphene. The method applied in this study is quite similar as the art of printing, through which an inhomogeneous, minor, and ultrafast strain due to FeNiCoTi SMA substrate surface relief was successfully printed on graphene, which can be quantitatively analyzed by monitoring the Raman spectra.

\section{Conclusion}

In summary, monolayer graphene was applied to characterize the inhomogeneous strain due to the surface relief in FeNiCoTi SMA substrate after phase transformation. The flat surface of the pristine substrate became wrinkled upon deep cryogenic treatment, during which loading an inhomogeneous tensile strain on the surface graphene. The 2D peak wavenumber of graphene shows a significant red shift rate of $\sim 31.2 \mathrm{~cm}^{-1} \%$ dependence on the tensile strain. Instead of AFM, the strain distribution and relief angle due to the surface relief in FeNiCoTi SMA substrate after phase transformation were quantitatively analyzed by using the graphene. The 2D peak of monolayer graphene exhibits ultrasensitivity to the strain, which is the key to its success in sensing the strain. 


\section{Acknowledgements}

This work was financially supported by the National Natural Science Foundation of China (Nos. 51071175, 51001119, 21106184, 21207144), Science Foundation of China University of Petroleum, Beijing (Nos. 2462013YJRC40, YJRC-2011-18) the National 973 program of China (2012CB619403), the Key Project of Chinese Ministry of Education (313055), and Thousand Talents Program of China.

\section{References}

[1] A. K. Geim, K. S. Novoselov, Nat. Mater. 2007, 6, 183.

[2] K. S. Novoselov, A. K. Geim, S. V. Morozov, D. Jiang, Y. Zhang, S. V. Dubonos, I. V. Grigorieva, A. A. Firsov, Science 2004, 306, 666.

[3] A. A. Balandin, S. Ghosh, W. Bao, I. Calizo, D. Teweldebrhan, F. Miao, C. N. Lau, Nano Lett. 2008, 8, 902.

[4] K. I. Bolotin, K. J. Sikes, Z. Jiang, M. Klima, G. Fudenberg, J. Hone, P. Kim, H. L. Stormer, Solid State Commun. 2008, 146, 351.

[5] L. M. Dai, Acc. Chem. Res. 2013, 46, 31.

[6] K. Kim, J. Y. Choi, T. Kim, S. H. Cho, H. J. Chung, Nature 2011, 479, 338.

[7] C. Lee, X. Wei, J. W. Kysar, J. Hone, Science 2008, 321, 385.

[8] M. H. Rummeli, C. G. Rocha, F. Ortmann, I. Ibrahim, H. Sevincli, F. Borrnert, J. Kunstmann, A. Bachmatiuk, M. Potschke, M. Shiraishi, M. Meyyappan, B. Buchner, S. Roche, G. Cuniberti, Adv. Mater. 2011, 23, 4471.

[9] Y. Q. Sun, Q. O. Wu, G. Q. Shi, Energy Environ. Sci. 2011, 4, 1113.

[10] X. Huang, Z. Y. Yin, S. X. Wu, X. Y. Qi, Q. Y. He, Q. C. Zhang, Q. Y. Yan, F. Boey, H. Zhang, Small 2011, 7, 1876.

[11] T. Zhang, Q. Z. Xue, S. Zhang, M. D. Dong, Nano Today 2012, 7, 180.

[12] S. Basu, P. Bhattacharyya, Sens. Actuators, B 2012, 173, 1.

[13] J. Liu, Z. Zeng, X. Cao, G. Lu, L.-H. Wang, Q.-L. Fan, W. Huang, H. Zhang, Small 2012, 8, 3517.

[14] C. Liu, F. Li, L. P. Ma, H. M. Cheng, Adv. Mater. 2010, 22, E28.

[15] Y.-M. Lin, C. Dimitrakopoulos, K. A. Jenkins, D. B. Farmer, H.-Y. Chiu, A. Grill, P. Avouris, Science 2010, 327, 662.

[16] M. Liu, X. Yin, E. Ulin-Avila, B. Geng, T. Zentgraf, L. Ju, F. Wang, X. Zhang, Nature 2011, 474, 64.

[17] K. S. Kim, Y. Zhao, H. Jang, S. Y. Lee, J. M. Kim, K. S. Kim, J.-H. Ahn, P. Kim, J.-Y. Choi, B. H. Hong, Nature 2009, 457, 706.

[18] S.-H. Bae, Y. Lee, B. K. Sharma, H.-J. Lee, J.-H. Kim, J.-H. Ahn, Carbon 2013, 51, 236.
[19] Y. Wang, R. Yang, Z. W. Shi, L. C. Zhang, D. X. Shi, E. Wang, G. Y. Zhang, ACS Nano 2011, 5, 3645.

[20] J. Zhao, C. L. He, R. Yang, Z. W. Shi, M. Cheng, W. Yang, G. B. Xie, D. M. Wang, D. X. Shi, G. Y. Zhang, Appl. Phys. Lett. 2012, 101, 063112.

[21] M. R. Falvo, G. J. Clary, R. M. Taylor, 2nd, V. Chi, F. P. Brooks, Jr., S. Washburn, R. Superfine, Nature 1997, 389, 582.

[22] Y. Lee, S. Bae, H. Jang, S. Jang, S. E. Zhu, S. H. Sim, Y. I. Song, B. H. Hong, J. H. Ahn, Nano Lett. 2010, 10, 490.

[23] X. W. Fu, Z. M. Liao, J. X. Zhou, Y. B. Zhou, H. C. Wu, R. Zhang, G. Y. Jing, J. Xu, X. S. Wu, W. L. Guo, D. P. Yu, Appl. Phys. Lett. 2011, 99, 213107.

[24] G. Gouadec, P. Colomban, Prog. Cryst. Growth Charact. Mater. 2007, $53,1$.

[25] R. J. Young, L. Gong, I. A. Kinloch, I. Riaz, R. Jalil, K. S. Novoselov, ACS Nano 2011, 5, 3079

[26] Y. Y. Wang, Z. H. Ni, T. Yu, Z. X. Shen, H. M. Wang, Y. H. Wu, W. Chen, A. T. S. Wee, J. Phys. Chem. C 2008, 112, 10637.

[27] Z. H. Ni, T. Yu, Y. H. Lu, Y. Y. Wang, Y. P. Feng, Z. X. Shen, ACS Nano 2008, 2, 2301

[28] G. Tsoukleri, J. Parthenios, K. Papagelis, R. Jalil, A. C. Ferrari, A. K. Geim, K. S. Novoselov, C. Galiotis, Small 2009, 5, 2397.

[29] D. Yoon, Y. W. Son, H. Cheong, Nano Lett. 2011, 11, 3227.

[30] T. Yu, Z. H. Ni, C. L. Du, Y. M. You, Y. Y. Wang, Z. X. Shen, J. Phys. Chem. C 2008, 112, 12602.

[31] S. M. Choi, S. H. Jhi, Y. W. Son, Phys. Rev. B 2010, 81, 081407.

[32] E. del Corro, A. O. de la Roza, M. Taravillo, V. G. Baonza, Carbon 2012, $50,4600$.

[33] M. A. Bissett, W. Izumida, R. Saito, H. Ago, Acs Nano 2012, 6, 10229.

[34] Z.-G. Yang, H.-S. Fang, J.-J. Wang, C.-M. Li, Y.-K. Zheng, Phys. Rev. B $1995,52,7879$.

[35] X. P. Lin, N. J. Gu, Y. Zhang, Z. W. Meng, X. L. Ma, Proc. Natl. Acad. Sci. U. S. A. 2002, 12, 766.

[36] M. Koyama, M. Murakami, K. Ogawa, T. Kikuchi, T. Sawaguchi, Mater. Trans. 2008, 49, 812.

[37] A. C. Ferrari, J. C. Meyer, V. Scardaci, C. Casiraghi, M. Lazzeri, F. Mauri, S. Piscanec, D. Jiang, K. S. Novoselov, S. Roth, A. K. Geim, Phys. Rev. Lett. 2006, 97, 187401.

[38] D. Graf, F. Molitor, K. Ensslin, C. Stampfer, A. Jungen, C. Hierold, L. Wirtz, Nano Lett. 2007, 7, 238.

\section{Supporting information}

Additional supporting information may be found in the online version of this article at the publisher's web site. 